\title{
The Heat Transfer Problem in a Rigid and Nonconvex Gray Body with Temperature Dependent Thermal Conductivity
}

\author{
Rogério Martins Saldanha da Gama ${ }^{1 *}$ and Maria Laura Martins-Costa ${ }^{2}$ \\ ${ }^{1}$ Mechanical Engineering Graduate Program (FEN) Universidade do Estado do Rio de Janeiro Rua São Francisco Xavier 524 $20550-013$, \\ Rio de Janeiro, Brazil \\ ${ }^{2}$ Laboratory of Theoretical and Applied Mechanics, Mechanical Engineering Graduate Program, Universidade Federal Fluminense, Rua \\ Passo da Pàtria, 156, 24210-240 Niterói, RJ, Brazil \\ *Corresponding author
}

\section{Article Info}

Keywords: Conduction-radiation, Fredholm equation, Non-convex gray bodies, Radiosity, Solution construction, Temperature-dependent conductivity. 2010 AMS: 25A12, 34G10, 34H15.

Received: 7 June 2018

Accepted: 4 January 2019 Available online: 20 April 2019

\begin{abstract}
This work studies coupled steady-state conduction-radiation heat transfer in a non-convex gray body when the thermal conductivity temperature-dependent. The gray-body assumption is an improvement with respect to the black body model, because in this model a portion of the incident radiant energy can be reflected from the body boundary. The problem is mathematically described by a nonlinear partial differential equation subjected to a nonlinear boundary condition involving a Fredholm operator which arises from the non-convexity of the body. In this problem the absolute temperature distribution is the unknown, as in the case of a black body. The Kirchhoff transformation is employed to linearize the partial differential equation, giving rise to new boundary conditions. The solution of the problem is constructed by a proposed iterative procedure, employing sequences that involve the temperature and the radiosity. The convergence is explicitly demonstrated. Besides, an error estimate, for each element, is presented. It is remarkable that the results obtained for black bodies are a particular case of this work. In other words, the results presented in reference [1] consists of a particular case of this paper, obtained when the emissivity is equal to one.
\end{abstract}

\section{Introduction}

Although many times the black body assumption may be a good approximation for engineering purposes, thermal radiant energy is emitted, absorbed and reflected by any real body. This article may be considered as a generalization of [1], aiming at a more realistic description, since real bodies boundaries actually reflect part of the incident radiant energy.

When the body is surrounded by a rarefied atmosphere or when the temperature levels are high, this thermal radiation exchange must be taken into account. As a first approximation, the body could be treated as a blackbody. Nevertheless, a blackbody [1] does not reflect thermal radiant energy and, many times, this fact does not allow a good mathematical description for bodies in which the reflection is not negligible. The simplest physical model for taking into account the reflection of thermal radiant energy is based on the gray body assumption. This assumption may be regarded as an improvement of the black body hypothesis, since it takes into account the reflection of thermal radiant energy. In other words, it is allowed that a part of the incident thermal radiant energy is not absorbed, but reflected.

When the body boundary is assumed to be opaque, a conduction heat transfer process takes place inside it, while a thermal radiation heat transfer takes place from/to the body boundary.

In this work the body boundary is assumed opaque and gray. In other words, it is assumed that, after reaching the boundary, the incident thermal radiant energy be divided into two parcels: one reflected and one absorbed (transferred to the inner part of the body by conduction heat transfer).

Non-convex bodies are characterized by a direct thermal radiant energy interchange among non-neighboring points on body boundary, since from all the energy coming from body boundary a portion of the emitted and a portion of the reflected thermal radiant energy reaches directly the same boundary. This effect causes more complex boundary conditions [2], [3]. 


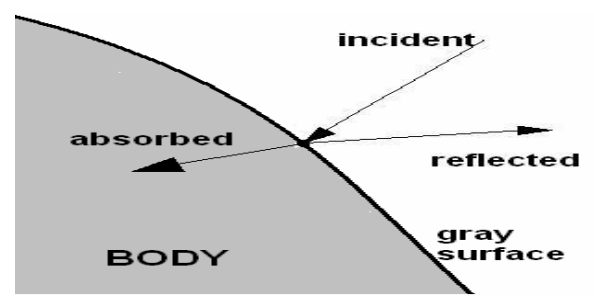

Figure 1.1: Scheme of a gray surface

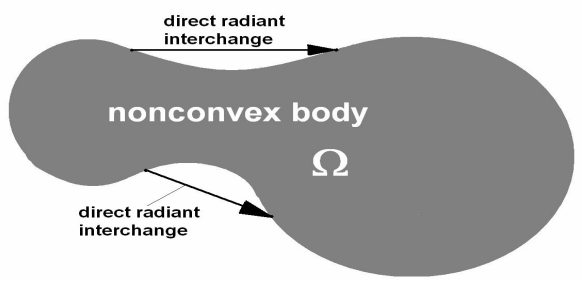

Figure 1.2: A nonconvex body. Radiant interchange among points on the boundary must be accounted for.

The mathematical modeling of the energy transfer process in a gray body is the subject of this work, in which it is assumed that the body may be non-convex and may have a temperature dependent thermal conductivity. The considered phenomenon can be found almost everywhere, since most bodies are not convex and possess conductivities that depend on the temperature.

Thermal radiation heat transfer and conduction heat transfer coupling occurs on the boundary of the body, since the normal heat flux must be continuous at any boundary point. So, an inherently nonlinear boundary condition must be satisfied.

In addition, conduction heat transfer is the energy transfer in the interior of the body. An elliptic partial differential equation [4] describes this steady-state conduction heat transfer. This partial differential equation becomes nonlinear in case of temperature-dependent thermal conductivity.

The thermal conductivity depends on the temperature [5], [6], [7], [8] and on the pressure [9] for all real materials. Under the rigid body assumption, in some cases, these dependences may be disregarded. However in some cases, it is important to consider a temperaturedependent thermal conductivity, since a body can experience large variations of conductivity [10],[11],[12],[13],[14].

Thermal radiant energy is emitted from all the points on the boundary of any real body because all the body points are at a temperature level above the absolute zero. In addition, there is a reflection of thermal radiant energy from all the points on the boundary which receive any thermal radiation.

This body may be represented by the bounded open set $\Omega$. When $\Omega$ is not convex, a part of the thermal radiant energy emitted and/or reflected from body boundary will reach, directly, the body, acting as an external temperature dependent heat source.

Assuming the body rigid, opaque and at rest, the energy transfer process inside $\Omega$ occurs solely by conduction heat transfer.

So, there is a coupling between a conduction heat transfer (inside $\Omega$ ) and a thermal radiant heat transfer (from/to $\partial \Omega$ ), concerning the energy transfer.

The main objective of this work is to construct the solution for the steady-state energy transfer process in a gray body with temperaturedependent thermal conductivity. Also existence and uniqueness of the solution of this solution are demonstrated.

Reference [1] may be considered as a particular case of the present work, which arises when the emissivity is equal to one (that means black body).

The mathematical description for a gray body is much more complex than the description for a black body, due to the boundary conditions. These boundary conditions must take into account that only a part of the incident thermal radiant energy is absorbed.

\section{Mathematical description}

The following partial differential equation [15], [16] describes the conduction heat transfer process inside $\Omega$, considering steady-state regimen

$$
\nabla \cdot(k \nabla T)+\dot{q}=0, \text { in } \Omega, \quad k=\hat{k}(T)
$$

in which $\dot{q}$ represents a known nonnegative field (an internal heat source) and $k$ is the thermal conductivity, a real positive-valued quantity depending on the temperature $T$.

Since $k=\hat{k}(T)$, equation (2.1) is not linear.

Considering a gray body, the thermal radiant energy emitted from a point on the boundary $\partial \Omega$ (per unit time and per unit area) is given by $\varepsilon \sigma\left|T^{3}\right| T$. In this expression, the Stefan-Boltzmann constant is represented by $\sigma$, while the emissivity is is represented by $\varepsilon$, being such that $0<\varepsilon \leq 1$. When $\varepsilon=1$ the black body hypothesis is assumed [15]. The incident thermal radiant energy at a given point $\mathbf{x} \in \partial \Omega$ (per unit time and per unit area) is given by [17],

$$
H=\hat{H}(\mathbf{x})=\int_{\mathbf{y} \in \partial \Omega} \hat{B}(\mathbf{y}) K d S+\hat{s}(\mathbf{x}), \quad K=\hat{K}(\mathbf{x}, \mathbf{y}) \quad \forall \mathbf{x} \in \partial \Omega
$$

in which $B=\hat{B}(\mathbf{x})$ is called radiosity (the sum of both emitted and reflected energy at a given point on the boundary). In equation (2.2) two effects are considered: the existence of an external thermal radiant source, $s=\hat{s}(\mathbf{x})$, and also the thermal radiation effect which emerges from points on $\partial \Omega$ and reaches the point $\mathbf{x} \in \partial \Omega$. The quantity $T=\hat{T}(\mathbf{x})$ in (2.2) stands for the absolute temperature at the point $\mathbf{x} \in \partial \Omega$. 
The kernel $K$ depends solely on the shape of $\Omega$ [17], being such that

$$
0 \leq \int_{\mathbf{y} \in \partial \Omega} K d S=\hat{\eta}(\mathbf{x}) \leq \beta<1, \quad \forall \mathbf{x} \in \partial \Omega
$$

in which $\beta$ is a constant.

The difference between the emitted and the absorbed thermal radiant energy is called thermal radiant heat flux from/to body boundary and is expressed in this work as $q_{R A D}$. This difference is exactly the difference between the radiosity and the incident thermal radiation. In other words,

$$
\begin{gathered}
q_{R A D}=B-H \text { on } \partial \Omega \\
B=\varepsilon \sigma\left|T^{3}\right| T+(1-\varepsilon) H
\end{gathered}
$$

in which $(1-\varepsilon)$ is the reflectance, since the body is opaque [17]. In a more convenient form

$$
B=\hat{B}(\mathbf{x}) \varepsilon \sigma|\hat{T}(\mathbf{x})|^{3} \hat{T}(\mathbf{x})+(1-\varepsilon) \int_{\mathbf{y} \in \partial \Omega} \hat{B}(\mathbf{y}) K d S+\hat{s}(\mathbf{x}), \quad \forall \mathbf{x} \in \partial \Omega
$$

Once $T$ is known, equation (2.4) is a Fredholm integral equation of the second kind.

The continuity of the normal heat flux across the boundary is ensured when the normal conduction heat flux equals the thermal radiant heat flux on $\partial \Omega$.

In consequence,

$$
\begin{array}{r}
\mathbf{q} \cdot \mathbf{n}=-k \nabla T \cdot \mathbf{n}=B-H, \text { on } \partial \Omega \\
\nabla \cdot(k \nabla T)+\dot{q}=0 \text { in } \Omega \\
-k \nabla T \cdot \mathbf{n}=B-H \text { on } \partial \Omega \\
B=\varepsilon \sigma\left|T^{3}\right| T+(1-\varepsilon) H \text { on } \partial \Omega \\
H=\mathfrak{I}[B]+\text { on } \partial \Omega
\end{array}
$$

in which the unknown is the absolute temperature field $T$ and the linear operator $\mathfrak{I}[B]$ is defined by

$$
\mathfrak{I}[B]=\int_{\mathbf{y} \in \partial \Omega} \hat{B}(\mathbf{y}) K d S, \quad \mathbf{x} \in \partial \Omega
$$

\section{The Kirchhoff transformation}

In order to employ the Kirchhoff transformation, the first step is the definition of an operator such that [18]

$$
\aleph[T]=\int_{0}^{t} \hat{T}(\xi) d \xi
$$

At this point one can take advantage of the fact that $\nabla \cdot(k \nabla T)=\nabla \cdot(\nabla \omega)$. In this case, the following differential equation is satisfied by the function $\omega=\aleph[T]$

$$
\nabla \cdot(\nabla \omega)+\dot{q}=0, \text { in } \Omega
$$

Once $\dot{q}$ is known, equation (3.2) is linear.

Considering any real material, the thermal conductivity $k$ is always a positive valued function. Actually, for any material, the thermal conductivity has a positive lower bound and the following inequality always holds

$$
\hat{k}(T) \geq \delta>0 \quad \text { for any } T, \quad \delta=\text { costant }
$$

So, the existence of the inverse of the Kirchhoff transformation, denoted by $T=\aleph^{-1}[\omega]$ is automatically ensured.

At this point it is convenient to use the Kirchhoff transformation to express the considered problem (problem (2.5)) in terms of the variable $\omega$

$$
\begin{array}{r}
\nabla \cdot(\nabla \omega)+\dot{q}=0 \text { in } \Omega \\
-\nabla \omega \cdot \mathbf{n}=B-H, \text { on } \partial \Omega \\
B=\varepsilon \sigma|T|^{3} T+(1-\varepsilon) H, \text { on } \partial \Omega \\
H=\mathfrak{I}[B]+s, \text { on } \partial \Omega \\
T=\mathfrak{\aleph}^{-1}[\omega]
\end{array}
$$


in which the unknown is $\omega$. It important to note that the determination of $\omega$ leads to the unique determination of the absolute temperature. Problem (3.3) may be also represented as

$$
\begin{array}{r}
\nabla \cdot(\nabla \omega)+\dot{q}=0 \text { in } \Omega \\
-\nabla \omega \cdot \mathbf{n}=\varepsilon \sigma|T|^{3} T-\varepsilon(\mathfrak{I}[B]+s) \text { on } \partial \Omega \\
B=\varepsilon \sigma|T|^{3} T+(1-\varepsilon)(\mathfrak{I}[B]+s) \text { on } \partial \Omega \\
T=\boldsymbol{\aleph}^{-1}[\omega] \\
\mathfrak{I}[B]=\int_{\mathbf{y} \in \partial \Omega} \hat{B}(\mathbf{y}) K d S, \quad \mathbf{x} \in \partial \Omega
\end{array}
$$

\section{Constructing the solution of problems with nonconvexity effects}

To reach the solution of problem $(2.5)$, the sequences $\left[\Phi^{1}, \Phi^{2}, \Phi^{3}, \ldots\right]$ and $\left[\Psi^{1}, \Psi^{2}, \Psi^{3}, \ldots\right]$ will be constructed. Their elements are obtained from

$$
\begin{array}{r}
\nabla \cdot\left(\nabla \Psi^{i+1}\right)+\dot{q}=0, \text { in } \Omega \\
-\left(\nabla \Psi^{i+1}\right) \cdot \mathbf{n}=\varepsilon \sigma\left|\Phi^{i+1}\right|^{3} \Phi^{i+1}-\varepsilon\left(\mathfrak{I}\left[\Theta^{i+1}\right]+s\right), \text { on } \partial \Omega \\
\Theta^{i+1}=\varepsilon \sigma\left|\Phi^{i+1}\right|^{3} \Phi^{i+1}+(1-\varepsilon)\left(\mathfrak{I}\left[\Theta^{i+1}\right]+s\right), \text { on } \partial \Omega \\
\Phi^{i}=\aleph^{-1}\left[\Psi^{i}\right] \\
i=1,2,3, \ldots
\end{array}
$$

in which

$$
\mathfrak{I}\left[\Theta^{i}\right]=\int_{\mathbf{y} \in \partial \Omega} \hat{\Theta}^{i}(\mathbf{y}) K d S, \quad \mathbf{x} \in \partial \Omega \quad, \quad i=1,2,3, \ldots
$$

and in such a way that

$$
\omega \equiv \lim _{i \rightarrow \infty} \Psi^{i} \quad \text { and } \quad T \equiv \lim _{i \rightarrow \infty} \Phi^{i}
$$

with $\Phi^{0} \equiv 0$ and $\Theta^{0} \equiv 0$. The existence of each element of the sequences is ensured from the coerciveness of the associated operator [19], [1].

Proposition 4.1. The solution of the problem (3.3), denoted by $T$ and such that $\omega=\aleph[T]$, is the limit of the sequence $\left[\Phi^{1}, \Phi^{2}, \Phi^{3}, \ldots\right]$ whose elements are obtained from (4.1), with $\Phi^{0} \equiv 0$ and $\Theta^{0} \equiv 0$.

Proof. (Part one).

The fields $\Phi^{1}$ and $\Psi^{1}$ are non negative.

Since $\dot{q} \geq 0$, the following nonempty subset $\partial \Omega^{-}$may be defined

$$
\begin{gathered}
\partial \Omega^{-} \equiv\left\{\mathbf{x} \in \partial \Omega \quad \text { such that }\left(\nabla \Phi^{1}\right) \cdot \mathbf{n} \leq 0\right\} \\
\inf _{\Omega} \Psi^{1}=\inf _{\partial \Omega} \Psi^{1}=\inf _{\partial \Omega^{-}} \Psi^{1}
\end{gathered}
$$

and, from the boundary conditions of (4.1),

$$
\begin{gathered}
\varepsilon \sigma\left|\Phi^{1}\right|{ }^{3} \Phi^{1}-\varepsilon\left(\mathfrak{I}\left[\Theta^{0}\right]+s\right) \geq 0 \text { on } \partial \Omega^{-} \Rightarrow \varepsilon \sigma\left|\Phi^{1}\right|{ }^{3} \Phi^{1}-\varepsilon s \geq 0 \text { on } \partial \Omega^{-} \\
\Phi^{1} \geq 0 \text { on } \partial \Omega^{-} \Rightarrow \inf _{\Omega^{-}} \Phi^{1} \geq 0
\end{gathered}
$$

Therefore, from (4.2),

$$
\Psi^{1}=\aleph\left[\Phi^{1}\right]=\int_{0}^{\Phi^{1}} \hat{k}(\xi) d \xi \geq 0 \text { on } \partial \Omega^{-} \Rightarrow \Psi^{1} \geq 0 \text { in } \Omega
$$

and, from (3.1),

$$
\Phi^{1} \geq 0 \text { in } \Omega
$$


Proof. (Part two).

The fields $\Phi^{i}$ and $\Psi^{i}$ are such that $\Phi^{i+1} \geq \Phi^{i}$ and $\Psi^{i+1} \geq \Psi^{i}$.

From (4.1) it comes that

$$
\begin{array}{r}
\nabla \cdot\left(\nabla\left(\Psi^{i+1}-\Psi^{i}\right)\right)=0, \text { in } \Omega \\
-\left(\nabla\left(\Psi^{i+1}-\Psi^{i}\right)\right) \cdot \mathbf{n}=\varepsilon \sigma\left|\Phi^{i+1}\right|^{3} \Phi^{i+1}-\varepsilon \sigma\left|\Phi^{i}\right|^{3} \Phi^{i}- \\
-\left(\varepsilon \mathfrak{I}\left[\Theta^{i}\right]+s\right)-\left(\varepsilon \mathfrak{I}\left[\Theta^{i-1}\right]+s\right), \text { on } \partial \Omega
\end{array}
$$

Now the nonempty subset $\partial \Omega_{i+1}^{-}$may be defined as

$$
\partial \Omega_{i+1}^{-} \equiv\left\{\mathbf{x} \in \partial \Omega \text { such that }\left(\nabla\left(\Phi^{i+1}-\Phi^{i}\right)\right) \cdot \mathbf{n} \leq 0\right\}
$$

such that

$$
\begin{gathered}
\inf _{\Omega}\left(\Psi^{i+1}-\Psi^{i}\right)=\inf _{\partial \Omega}\left(\Psi^{i+1}-\Psi^{i}\right)=\inf _{\partial \Omega_{i+1}^{-}}\left(\Psi^{i+1}-\Psi^{i}\right) \\
\varepsilon \sigma\left|\Phi^{i+1}\right|^{3} \Phi^{i+1}-\varepsilon \sigma\left|\Phi^{i}\right|^{3} \Phi^{i} \geq \varepsilon\left(\mathfrak{I}\left[\Theta^{i}\right]-\mathfrak{I}\left[\Theta^{i-1}\right]\right) \text { on } \partial \Omega_{i+1}^{-}
\end{gathered}
$$

Therefore, considering that (4.3) is verified and $\Phi^{0} \equiv 0$,

$$
\Phi^{1}=\varepsilon \sigma\left|\Phi^{1}\right|^{3} \Phi^{1}+(1-\varepsilon) s \geq 0 \quad \text { on } \partial \Omega \quad \Rightarrow \quad \mathfrak{I}\left[\Theta^{1}\right] \geq \mathfrak{I}\left[\Theta^{0}\right] \text { on } \partial \Omega
$$

Thus,

$$
\begin{aligned}
& \Phi^{2}-\Phi^{1} \geq 0 \text { on } \partial \Omega_{2}^{-} \\
& \Psi^{2}-\Psi^{1} \geq 0 \text { on } \partial \Omega_{2}^{-}
\end{aligned}
$$

Hence, from (4.5)

$$
\inf _{\Omega}\left(\Psi^{2}-\Psi^{1}\right)=\inf _{\partial \Omega}\left(\Psi^{2}-\Psi^{1}\right)=\inf _{\partial \Omega_{2}^{-}}\left(\Psi^{2}-\Psi^{1}\right) \Rightarrow \Psi^{2} \geq \Psi^{1} \quad \text { in } \Omega
$$

and, consequently

$$
\Phi^{2} \geq \Phi^{1} \text { on } \partial \Omega \text { and } \Theta^{2} \geq \Theta^{1} \text { on } \partial \Omega
$$

The above procedure can be repeated in order to lead to the conclusion that $\Psi^{3} \geq \Psi^{2}$ in $\Omega$, that $\Phi^{3} \geq \Phi^{2}$ on $\partial \Omega$ and also that $\Theta^{3} \geq \Theta^{2}$ on $\partial \Omega$. So, the following may be written,

$$
\Psi^{i+1} \geq \Psi^{i} \text { in } \Omega, \quad \Phi^{i+1} \geq \Phi^{i} \text { on } \partial \Omega \text { and } \Theta^{i+1} \geq \Theta^{i} \text { on } \partial \Omega
$$

The above result (2.3) is very important because it allows ensuring that the sequences $\left[\Psi^{1}, \Psi^{2}, \Psi^{3}, \ldots\right],\left[\Phi^{1}, \Phi^{2}, \Phi^{3}, \ldots\right]$ and $\left[\Theta^{1}, \Theta^{2}, \Theta^{3}, \ldots\right]$ are non decreasing.

Proof. (Part three). Convergence of the sequence $\left[\Theta^{1}, \Theta^{2}, \Theta^{3}, \ldots\right]$.

Now one should prove that the sequence $\left[\Theta^{1}, \Theta^{2}, \Theta^{3}, \ldots\right]$ converges in the norm of $L^{1}(\partial \Omega)$. To achieve this objective problem (4.4) is considered, allowing to conclude that

$$
\int_{\partial \Omega}\left(\varepsilon \sigma\left|\Phi^{i+1}\right|^{3} \Phi^{i+1}-\varepsilon \sigma\left|\Phi^{i}\right|^{3} \Phi^{i}\right) d S=\int_{\mathbf{x} \in \partial \Omega} \int_{\mathbf{y} \in \partial \Omega} \varepsilon\left(\hat{\Theta}^{i}(\mathbf{y})-\hat{\Theta}^{i-1}(\mathbf{y})\right) K d S
$$

Considering the following property of the kernel $K(\mathbf{x}, \mathbf{y}) \equiv K(\mathbf{y}, \mathbf{x})$, equation (2.3) enables to write

$$
\int_{\partial \Omega} \varepsilon \sigma\left(\left|\Phi^{i+1}\right|^{3} \Phi^{i+1}-\left|\Phi^{i}\right|^{3} \Phi^{i}\right) d S \leq \beta \int_{\partial \Omega} \varepsilon\left(\Theta^{i}-\Theta^{i-1}\right) d S
$$

In addition, from (4.1)

$$
\Theta^{i}-\Theta^{i-1}=\varepsilon \sigma\left(\left|\Phi^{i}\right|^{3} \Phi^{i}-\left|\Phi^{i-1}\right|^{3} \Phi^{i-1}\right)+(1-\varepsilon)\left(\mathfrak{I}\left[\Theta^{i-1}\right]-\mathfrak{I}\left[\Theta^{i-2}\right]\right)
$$


Equation (4.8) enables to write

$$
\int_{\partial \Omega}\left(\Theta^{i}-\Theta^{i-1}\right) d S=\int_{\partial \Omega} \varepsilon \sigma\left(\left|\Phi^{i}\right|^{3} \Phi^{i}-\left|\Phi^{i-1}\right|^{3} \Phi^{i-1}\right) d S+\int_{\partial \Omega}(1-\varepsilon)\left(\mathfrak{I}\left[\Theta^{i-1}\right]-\mathfrak{I}\left[\Theta^{i-2}\right]\right) d S
$$

and, from (2.3), the following inequality comes

$$
\int_{\partial \Omega}\left(\Theta^{i}-\Theta^{i-1}\right) d S \leq \int_{\partial \Omega} \varepsilon \sigma\left(\left|\Phi^{i}\right|^{3} \Phi^{i}-\left|\Phi^{i-1}\right|^{3} \Phi^{i-1}\right) d S+\beta \int_{\partial \Omega}(1-\varepsilon)\left(\Theta^{i-1}-\Theta^{i-2}\right) d S
$$

Now, taking equation (4.7) into account, the following holds

$$
\int_{\partial \Omega}\left(\Theta^{i}-\Theta^{i-1}\right) d S \leq \beta \int_{\partial \Omega} \varepsilon\left(\Theta^{i-1}-\Theta^{i-2}\right) d S+\beta \int_{\partial \Omega}(1-\varepsilon)\left(\Theta^{i-1}-\Theta^{i-2}\right) d S
$$

and, hence,

$$
\int_{\partial \Omega}\left(\Theta^{i}-\Theta^{i-1}\right) d S \leq \beta \int_{\partial \Omega}\left(\Theta^{i-1}-\Theta^{i-2}\right) d S
$$

Since $\Theta^{i+1} \geq \Theta^{i}$ there is a contraction, ensuring the convergence of the sequence $\left[\Theta^{1}, \Theta^{2}, \Theta^{3}, \ldots\right]$ in $L^{1}(\partial \Omega)$. In addition, for any integer $i \geq 0$

$$
\left\|\Theta^{i+1}-\Theta^{i}\right\|_{L^{1}(\partial \Omega)}=\int_{\partial \Omega}\left(\Theta^{i+1}-\Theta^{i}\right) d S \leq \beta^{i} \int_{\partial \Omega} \Theta^{1} d S=\bar{M} \beta^{i}
$$

in which $\bar{M}$ is a positive constant. Therefore it follows that

$$
\Theta^{\infty} \equiv \lim _{i \rightarrow \infty} \Theta^{i} \text { on } \partial \Omega, \text { with } \Theta^{\infty} \in L^{1}(\partial \Omega)
$$

Proof. (Part four). Convergence of the sequence $\left[\Phi^{1}, \Phi^{2}, \Phi^{3}, \ldots\right]$ on the boundary.

At this point the convergence of the sequence $\left[\Phi^{1}, \Phi^{2}, \Phi^{3}, \ldots\right]$ can be demonstrated. Aiming to this, one can consider again (4.4), to write

$$
\int_{\partial \Omega}\left(\varepsilon \sigma\left|\Phi^{i+1}\right|^{3} \Phi^{i+1}-\varepsilon \sigma\left|\Phi^{i}\right|^{3} \Phi^{i}\right) d S=\int_{\mathbf{x} \in \partial \Omega} \int_{\mathbf{y} \in \partial \Omega} \varepsilon\left(\hat{\Theta}^{i}(\mathbf{y})-\hat{\Theta}^{i-1}(\mathbf{y})\right) K d S
$$

Inequality (2.3) allows to write

$$
\int_{\partial \Omega}\left(\varepsilon \sigma\left|\Phi^{i+1}\right|^{3} \Phi^{i+1}-\varepsilon \sigma\left|\Phi^{i}\right|^{3} \Phi^{i}\right) d S \leq \beta \int_{\partial \Omega} \varepsilon\left(\Theta^{i}-\Theta^{i-1}\right) d S
$$

giving rise to

$$
\int_{\partial \Omega} \sigma\left(\left|\Phi^{i+1}\right|^{3} \Phi^{i+1}-\left|\Phi^{i}\right|^{3} \Phi^{i}\right) d S \leq \bar{M} \beta^{i}\left(\frac{1-\varepsilon}{1-\beta \varepsilon}\right)^{i-1} \quad, \quad i=1,2,3, \ldots
$$

In addition, considering that $\Phi^{i+1} \geq \Phi^{i} \geq 0$, it comes that

$$
\left(\left\|\Phi^{i+1}-\Phi^{i}\right\|_{L^{4}(\partial \Omega)}\right)^{4}=\int_{\partial \Omega}\left|\Phi^{i+1}-\Phi^{i}\right|^{4} d S \leq \bar{M} \beta^{i}\left(\frac{1-\varepsilon}{1-\beta \varepsilon}\right)^{i-1} \quad, \quad i=1,2,3, \ldots
$$

The convergence is quite obvious for $\beta=0$. According to reference [20] there is a contraction for $0<\beta<1$. So, since $\Phi^{i}$ must belong to the Banach space $L^{4}(\partial \Omega)$, the sequence $\left[\Phi^{1}, \Phi^{2}, \Phi^{3}, \ldots\right]$ converges in this space. This allows concluding that

$$
\Phi^{\infty} \equiv \lim _{i \rightarrow \infty} \Phi^{i} \text { on } \partial \Omega, \text { with } \Phi^{\infty} \in L^{4}(\partial \Omega)
$$


Proof. (Part five). The limit of the sequence $\left[\Psi^{1}, \Psi^{2}, \Psi^{3}, \ldots\right]$ is a solution of (4.4).

From problem (4.1), the limit of the sequence $\left[\Psi^{1}, \Psi^{2}, \Psi^{3}, \ldots\right]$, expressed as by $\Psi^{\infty}$, may be represented as the solution of

$$
\begin{array}{r}
\nabla \cdot\left(\nabla \Psi^{\infty}\right)+\dot{q}=0 \text { in } \Omega \\
-\left(\nabla \Psi^{\infty}\right) \cdot \mathbf{n}=\varepsilon \sigma\left|\aleph^{-1}\left[\Psi^{\infty}\right]\right|^{3}\left(\aleph^{-1}\left[\Psi^{\infty}\right]\right)-E \text { on } \partial \Omega
\end{array}
$$

in which $E=\hat{E}(\mathbf{x})$ is a known function defined on the boundary as follows

$$
E=\varepsilon\left(\mathfrak{I}\left[\Theta^{\infty}\right]+s\right)=\varepsilon\left(\int_{\mathbf{y} \in \partial \Omega} \hat{\Theta}^{\infty} K d S+s\right) \text { on } \partial \Omega
$$

Therefore it may be concluded that $\omega \equiv \Psi^{\infty}$, limit of the sequence $\left[\Psi^{1}, \Psi^{2}, \Psi^{3}, \ldots\right]$, is a solution of problem (3.3), thus demonstating the solution existence. The uniqueness of the solution is demonstrated in the next step.

Proof. (Part six). The limit of the sequence corresponds to the unique solution of (3.3).

To demonstrate that the limit of the sequence $\left[\Psi^{1}, \Psi^{2}, \Psi^{3}, \ldots\right]$ is the unique solution of (3.4), one can assume the existence of a field $u$, which is supposed different from $\Psi^{\infty}$, and satisfies

$$
\begin{aligned}
& \nabla \cdot(\nabla u)+\dot{q}=0 \text { in } \Omega \\
& -\nabla u \cdot \mathbf{n}=\varepsilon \sigma|v|^{3} v-\varepsilon(\mathfrak{I}[z]+s) \text { on } \partial \Omega \\
& z=\varepsilon \sigma|v|^{3} v+(1-\varepsilon)(\mathfrak{I}[z]+s) \text { on } \partial \Omega \\
& v=\aleph^{-1}[u] \\
& \mathfrak{I}[z]=\int_{\mathbf{y} \in \partial \Omega} \hat{z}(\mathbf{y}) K d S, \quad \mathbf{x} \in \partial \Omega
\end{aligned}
$$

Defining the nonempty subset $\partial \Omega_{u}^{-}$as

$$
\partial \Omega_{u}^{-} \equiv\{\mathbf{x} \in \partial \Omega \text { such that }(\nabla u) \cdot \mathbf{n} \leq 0\}
$$

And, reminding that $(\nabla v) \cdot \mathbf{n} \leq 0$ on $\partial \Omega_{u}^{-}$, it comes that

$$
\begin{gathered}
\inf _{\Omega} u=\inf _{\partial \Omega} u=\inf _{\partial \Omega_{u}^{-}} u \quad \text { and } \quad \inf _{\Omega} v=\inf _{\partial \Omega} v=\inf _{\partial \Omega_{u}^{-}} v \\
\varepsilon \sigma|v|^{3} v-\varepsilon(\mathfrak{I}[z]+s) \geq 0 \text { on } \partial \Omega_{u}^{-} \\
z=\varepsilon \sigma|v|^{3} v+(1-\varepsilon)(\mathfrak{I}[z]+s) \text { on } \partial \Omega_{u}^{-} \\
v=\aleph^{-1}[u]
\end{gathered}
$$

Since the quantity $s$ is nonnegative and (4.11) is verified, (4.12) leads to

$$
\begin{array}{r}
\varepsilon \sigma|v|^{3} v-\varepsilon(\mathfrak{I}[z]+s) \geq 0 \text { on } \partial \Omega_{u}^{-} \quad \text { and } \quad z=\varepsilon \sigma|v|^{3} v+(1-\varepsilon)(\mathfrak{I}[z]+s) \\
\Rightarrow \quad \sigma|v|^{3} v \geq z \text { on } \partial \Omega_{u}^{-} \Rightarrow \sigma|v|^{3} v \geq(\mathfrak{I}[z]+s) \text { on } \partial \Omega_{u}^{-} \\
\Rightarrow \sigma\left|\inf _{\partial \Omega_{u}^{-}} v\right|^{3} \inf _{\partial \Omega_{u}^{-}} v \geq \inf _{\partial \Omega_{u}^{-}} \Rightarrow \quad \Rightarrow \quad \sigma\left|\inf _{\partial \Omega} v\right|^{3} \inf _{\partial \Omega} v \geq \inf _{\partial \Omega} z
\end{array}
$$

In addition, from (4.12) and (4.13), it comes that

$$
\inf _{\partial \Omega} z \geq \varepsilon \inf _{\partial \Omega} z+(1-\varepsilon) \geq \inf _{\partial \Omega}(\mathfrak{I}[z])
$$

Since,

$$
\inf _{\partial \Omega}(\mathfrak{I}[z]) \geq \hat{\eta}(\mathbf{x}) \inf _{\partial \Omega} z \quad \text { for some } \mathbf{x} \in \partial \Omega
$$

from (4.14), one can state that

$$
\inf _{\partial \Omega} z \geq \inf _{\partial \Omega}(\mathfrak{I}[z]) \geq \hat{\eta}(\mathbf{x}) \inf _{\partial \Omega} z \quad \text { for some } \mathbf{x} \in \partial \Omega
$$

in which $0 \leq \hat{\eta}(\mathbf{x})<\beta$. Therefore, 


$$
\inf _{\partial \Omega} z \geq 0 \Rightarrow \inf _{\partial \Omega} v \geq 0 \Rightarrow \inf _{\Omega} v \geq 0
$$

Now, considering (3.1), the following holds

$$
\inf _{\partial \Omega} v \geq 0 \Rightarrow \inf _{\partial \Omega} u \geq 0 \Rightarrow v \geq 0 \text { in } \Omega \Rightarrow u \geq 0 \text { in } \Omega
$$

Combining (4.10) with (4.1), gives rise to

$$
\begin{array}{r}
\nabla \cdot\left(\nabla\left(u-\Psi^{i+1}\right)\right)=0 \quad \text { in } \Omega \\
-\left(\nabla\left(u-\Psi^{i+1}\right)\right) \cdot \mathbf{n}=\varepsilon \sigma\left(|v|^{3} v-\left|\Phi^{i+1}\right|^{3} \Phi^{i+1}\right)-\varepsilon\left(\mathfrak{I}[z]-\mathfrak{I}\left[\Theta^{i}\right]\right) \quad \text { on } \partial \Omega \\
z-\Theta^{i+1}=\varepsilon \sigma\left(|v|^{3} v-\left|\Phi^{i+1}\right|^{3} \Phi^{i+1}\right)+(1-\varepsilon)\left(\mathfrak{I}[z]-\mathfrak{I}\left[\Theta^{i}\right]\right) \quad \text { on } \partial \Omega \\
v=\mathfrak{\aleph}^{-1}[u], \Phi^{i}=\mathfrak{\aleph}^{-1}\left[\Psi^{i}\right] \\
\mathfrak{I}[z]=\int_{\mathbf{y} \in \partial \Omega} \hat{z}(\mathbf{y}) K d S, \quad \mathbf{x} \in \partial \Omega \\
\mathfrak{I}\left[\Theta^{i}\right]=\int_{\mathbf{y} \in \partial \Omega} \hat{\Theta}^{i}(\mathbf{y}) K d S, \quad \mathbf{x} \in \partial \Omega
\end{array}
$$

Defining the nonempty subset $\partial \Omega_{u}^{i+1}$ as

$$
\partial \Omega_{u}^{i+1} \equiv\left\{\mathbf{x} \in \partial \Omega \text { such that } \nabla\left(u-\Psi^{i+1}\right) \cdot \mathbf{n} \leq 0\right\}
$$

it comes that

$$
\inf _{\Omega}\left(u-\Psi^{i+1}\right)=\inf _{\partial \Omega}\left(u-\Psi^{i+1}\right)=\inf _{\partial \Omega_{u}^{i+1}}\left(u-\Psi^{i+1}\right)
$$

So,

$$
u \geq \Psi^{i+1} \quad \text { on } \partial \Omega_{u}^{i+1} \Rightarrow u \geq \Psi^{i+1} \quad \text { in } \Omega \text { for any } i
$$

and, accounting for (3.1) it comes that

$$
u \geq \Psi^{i+1} \quad \text { in } \Omega \quad v \geq \Phi^{i+1} \text { in } \Omega \text { for any } i
$$

Since

$$
\varepsilon \sigma\left(|v|^{3} v-\left|\Phi^{i+1}\right|^{3} \Phi^{i+1}\right)-\varepsilon\left(\mathfrak{I}[z]-\mathfrak{I}\left[\Theta^{i}\right]\right) \geq 0 \quad \text { on } \partial \Omega_{u}^{i+1}
$$

and (4.6) and (4.16) hold, the following must also hold

$$
\begin{gathered}
z \geq \Theta^{i} \text { on } \partial \Omega \Rightarrow v \geq \Psi^{i+1} \text { on } \partial \Omega_{u}^{i+1} \quad \Rightarrow \\
\Rightarrow \quad u \geq \Psi^{i+1} \text { on } \partial \Omega_{u}^{i+1} \quad \Rightarrow \quad u \geq \Psi^{i+1} \text { in } \Omega \quad \Rightarrow \\
\Rightarrow v \geq \Phi^{i+1} \text { in } \Omega \Rightarrow z \geq \Theta^{i+1} \text { on } \partial \Omega \Rightarrow \ldots
\end{gathered}
$$

Therefore, the following result can be ensured

$$
z \geq \Theta^{i} \text { on } \partial \Omega \Rightarrow u \geq \Psi^{i+1} \quad \text { in } \Omega \quad \text { and } \quad v \geq \Phi^{i+1} \quad \text { in } \Omega \quad \text { for any } i
$$

Since $\Theta^{0} \equiv 0$ on $\partial \Omega,(4.17)$ yields

$$
u \geq \Psi^{\infty} \text { and } \quad v \geq \Phi^{\infty} \text { in } \Omega \text { and } z \geq \Theta^{\infty} \text { on } \partial \Omega
$$

The existence of the limits $\Psi^{\infty}, \Phi^{\infty}$ and $\Theta^{\infty}$ allows to write (4.15), for $i \rightarrow \infty$ 


$$
\begin{array}{r}
\nabla \cdot\left(\nabla\left(u-\Psi^{\infty}\right)\right)=0 \quad \text { in } \Omega \\
-\left(\nabla\left(u-\Psi^{\infty}\right)\right) \cdot \mathbf{n}=\varepsilon \sigma\left(|v|^{3} v-\left|\Phi^{\infty}\right|^{3} \Phi^{\infty}\right)-\varepsilon\left(\mathfrak{I}[z]-\mathfrak{I}\left[\Theta^{\infty}\right]\right) \quad \text { on } \partial \Omega \\
z-\Theta^{\infty}=\varepsilon \sigma\left(|v|^{3} v-\left|\Phi^{\infty}\right|^{3} \Phi^{\infty}\right)+(1-\varepsilon)\left(\mathfrak{I}[z]-\mathfrak{I}\left[\Theta^{\infty}\right]\right) \quad \text { on } \partial \Omega \\
v=\mathfrak{\aleph}^{-1}[u], \Phi^{i}=\aleph^{-1}\left[\Psi^{\infty}\right] \\
\mathfrak{I}[z]=\int_{\mathbf{y} \in \partial \Omega} \hat{z}(\mathbf{y}) K d S, \quad \mathbf{x} \in \partial \Omega \\
\mathfrak{I}\left[\Theta^{\infty}\right]=\int_{\mathbf{y} \in \partial \Omega} \hat{\Theta^{\infty}}(\mathbf{y}) K d S, \quad \mathbf{x} \in \partial \Omega
\end{array}
$$

Problem (4.19) allows to write

$$
\begin{array}{r}
\int_{\partial \Omega}\left\{\varepsilon \sigma\left(|v|^{3} v-\left|\Phi^{\infty}\right|^{3} \Phi^{\infty}\right)-\varepsilon\left(\mathfrak{I}[z]-\mathfrak{I}\left[\Theta^{\infty}\right]\right)\right\} d S=0 \\
\int_{\partial \Omega}\left(z-\Theta^{\infty}\right) d S=\int_{\partial \Omega}\left\{\varepsilon \sigma\left(|v|^{3} v-\left|\Phi^{\infty}\right|^{3} \Phi^{\infty}\right)-\varepsilon\left(\mathfrak{I}[z]-\mathfrak{I}\left[\Theta^{\infty}\right]\right)\right\} d S
\end{array}
$$

in which

$$
\int_{\partial \Omega}\left(\mathfrak{I}[z]-\mathfrak{I}\left[\Theta^{\infty}\right]\right) d S=\int_{\mathbf{x} \in \partial \Omega}\left(\int_{\mathbf{y} \in \partial \Omega}(\hat{z}(\mathbf{y}) \hat{\Theta}(\mathbf{y}) K) d S\right) d S \leq \beta \int_{\partial \Omega}(z-\Theta) d S
$$

Therefore, combining (4.20) with (4.21)

$$
\int_{\partial \Omega}\left(z-\Theta^{\infty}\right) d S \leq \beta \int_{\partial \Omega}\left(z-\Theta^{\infty}\right) d S
$$

From (4.18) and (4.22), since $0 \leq \beta<1$, it can be concluded that

$$
z=\Theta^{\infty} \text { on } \partial \Omega
$$

Hence,

$$
v=\Phi^{\infty} \quad \text { in } \Omega
$$

and the difference $u-\Psi^{\infty}$ is zero everywhere (that means $u=\Psi^{\infty}$ in $\Omega$ ), since it is the unique solution of the well-known Dirichlet problem [4]

$$
\begin{gathered}
\nabla \cdot\left(\nabla\left(u-\Psi^{\infty}\right)\right)=0 \quad \text { in } \Omega \\
u-\Psi^{\infty}=0 \text { on } \partial \Omega
\end{gathered}
$$

Therefore, the temperature $T$, which is the solution of (2.5), is such that

$$
T \equiv \lim _{i \rightarrow \infty} \Psi^{i}=\Psi^{\infty} \quad \text { in } \Omega
$$

while $\omega(\omega=\aleph[T])$ is given by $\Psi^{\infty}$ and $B$ is given by $\Theta^{\infty}$.

\section{On the difference between the solution and the i-th element of the sequence}

At this section the goal is present a tool that enables verifying whether the element $\Phi^{i}$ is sufficiently precise for simulation purposes. Aiming at this goal, one can consider problem (4.15), replacing $u$ by $\omega, v$ by $T$ and $z$ by $B$, as follows

$$
\begin{aligned}
& \nabla \cdot\left(\nabla\left(\omega-\Psi^{i+1}\right)\right)=0 \quad \text { in } \Omega \\
& -\left(\nabla\left(\omega-\Psi^{i+1}\right)\right) \cdot \mathbf{n}=\varepsilon \sigma\left(|T|^{3} T-\left|\Phi^{i+1}\right|{ }^{3} \Phi^{i+1}\right)-\varepsilon\left(\mathfrak{I}[B]-\mathfrak{I}\left[\Theta^{i}\right]\right) \quad \text { on } \partial \Omega \\
& B-\Theta^{i+1}=\varepsilon \sigma\left(|T|^{3} T-\left|\Phi^{i+1}\right|{ }^{3} \Phi^{i+1}\right)+(1-\varepsilon)\left(\mathfrak{I}[B]-\mathfrak{I}\left[\Theta^{i}\right]\right) \quad \text { on } \partial \Omega \\
& T=\mathfrak{\aleph}^{-1}[\omega], \Phi^{i}=\mathfrak{\aleph}^{-1}\left[\Psi^{i}\right] \\
& \mathfrak{I}[B]=\int_{\mathbf{y} \in \partial \Omega} \hat{B}(\mathbf{y}) K d S, \quad \mathbf{x} \in \partial \Omega
\end{aligned}
$$


Since $\nabla \cdot\left(\nabla\left(\omega-\Psi^{i+1}\right)\right)$ is zero everywhere, the boundary condition yields

$$
\int_{\partial \Omega} \varepsilon\left(\sigma|T|^{3} T-\left|\Phi^{i+1}\right|^{3} \Phi^{i+1}\right) d S=\int_{\mathbf{x} \in \partial \Omega}\left(\int_{\mathbf{y} \in \partial \Omega} \varepsilon\left(\hat{B}(\mathbf{y}) \hat{\Theta}^{i}(\mathbf{y}) K\right) d S\right) d S
$$

and, therefore,

$$
\int_{\partial \Omega} \varepsilon\left(\sigma|T|^{3} T-\left|\Phi^{i+1}\right|^{3} \Phi^{i+1}\right) d S \leq \beta \int_{\partial \Omega} \varepsilon\left(B-\Theta^{i}\right) d S
$$

Since

$$
\int_{\partial \Omega} \varepsilon\left(B-\Theta^{i}\right) d S=\int_{\partial \Omega} \varepsilon \sigma\left(|T|^{3} T-\left|\Phi^{i+1}\right|^{3} \Phi^{i+1}\right) d S+\int_{\partial \Omega}(1-\varepsilon)\left(\mathfrak{I}[B]-\mathfrak{I}\left[\Theta^{i}\right]\right) d S
$$

Combining (2.3), (5.1) and (5.2), one may write

$$
\begin{array}{r}
\int_{\partial \Omega} \varepsilon\left(\sigma|T|^{3} T-\sigma\left|\Phi^{i+1}\right|^{3} \Phi^{i+1}\right) d S \leq \beta \int_{\partial \Omega} \varepsilon\left(B-\Theta^{i}\right) d S+\beta \int_{\partial \Omega} \varepsilon\left(\Theta^{i+1}-\Theta^{i}\right) d S \\
\int_{\partial \Omega}\left(B-\Theta^{i+1}\right) d S \leq \int_{\partial \Omega} \varepsilon \sigma\left(|T|^{3} T-\left|\Phi^{i+1}\right|^{3} \Phi^{i+1}\right) d S+ \\
+\beta \int_{\partial \Omega}(1-\varepsilon)\left(\Theta^{i+1}-\Theta^{i}\right) d S+\beta \int_{\partial \Omega}(1-\varepsilon)\left(B-\Theta^{i}+1\right) d S
\end{array}
$$

Inequalities (5.3) allow to write

$$
\int_{\partial \Omega}\left(B-\Theta^{i+1}\right) d S \leq \frac{\beta}{1-\beta} \int_{\partial \Omega}\left(\Theta^{i+1}-\Theta^{i}\right) d S
$$

then, from (4.9)

$$
\int_{\partial \Omega}\left(B-\Theta^{i+1}\right) d S \leq \frac{\beta}{1-\beta} \int_{\partial \Omega}\left(\Theta^{i+1}-\Theta^{i}\right) d S \leq \frac{\beta^{i+1}}{1-\beta} \int_{\partial \Omega} \Theta^{1} d S
$$

From (4.1), when $i=1$, it comes that

$$
\int_{\Omega} \dot{q} d V=\int_{\partial \Omega}\left(\varepsilon \sigma\left|\Phi^{1}\right|^{3} \Phi^{1}-\varepsilon s\right) d S+\int_{\partial \Omega}\left(\Theta^{1}-s\right) d S
$$

Therefore,

$$
\int_{\partial \Omega}\left(B-\Theta^{i}\right) d S \leq \frac{\beta^{i}}{1-\beta}\left(\int_{\Omega} \dot{q} d V+\int_{\partial \Omega} s d S\right)
$$

and, from (5.1),

$$
\int_{\partial \Omega}\left(\sigma|T|^{3} T-\sigma\left|\Phi^{i}\right|^{3} \Phi^{i}\right) d S \leq \frac{\beta^{i}}{1-\beta}\left(\int_{\Omega} \dot{q} d V+\int_{\partial \Omega} s d S\right)
$$

Since $T \geq \Phi^{i}$, inequality (5.5) yields

$$
\left\|T-\Phi^{i}\right\|_{L^{4}(\partial \Omega)} \leq C \beta^{i / 4}
$$

in which \|\|$_{L^{4}(\partial \Omega)}$ represents the usual $L^{4}(\partial \Omega)$ norm and $C$ is a constant defined as

$$
C=\left(\frac{1}{1-\beta}\left(\int_{\Omega} \dot{q} d V+\int_{\partial \Omega} s d S\right)\right)^{1 / 4}
$$

If the kernel $K$ is bounded, estimate (5.6) may be improved. In this case, a nonempty subset $\partial \Omega_{++}^{i+1}$ can be defined as

$$
\partial \Omega_{++}^{i+1} \equiv\left\{\mathbf{x} \in \partial \Omega \text { such that } \nabla\left(\omega-\Psi^{i+1}\right) \cdot \mathbf{n} \leq 0\right\}
$$

Therefore, 
28

Journal of Mathematical Sciences and Modelling

$$
\sup _{\Omega}\left(\omega-\Psi^{i+1}\right)=\sup _{\partial \Omega}\left(\omega-\Psi^{i+1}\right)=\sup _{\partial \Omega_{++}^{i+1}}\left(\omega-\Psi^{i+1}\right)
$$

and

$$
\varepsilon\left(\sigma|T|^{3} T-\sigma\left|\Phi^{i+1}\right|^{3} \Phi^{i+1}\right) \leq \int_{\mathbf{y} \in \partial \Omega}\left(\hat{B}(\mathbf{y})-\hat{\Theta}^{i}(\mathbf{y}) K\right) d S \quad \text { on } \partial \Omega_{++}^{i+1}
$$

Considering that the kernel is bounded and $\varepsilon>0$, one may conclude that

$$
\sigma|T|^{3} T-\sigma\left|\Phi^{i+1}\right|^{3} \Phi^{i+1} \leq \sup _{\mathbf{x} \in \partial \Omega, \mathbf{y} \in \partial \Omega} K \int_{\mathbf{y} \in \partial \Omega}\left(\hat{B}(\mathbf{y})-\hat{\Theta}^{i}(\mathbf{y})\right) d S \quad \text { on } \partial \Omega_{++}^{i+1}
$$

and, accounting for inequation (5.4)

$$
|T|^{3} T-\left|\Phi^{i+1}\right|^{3} \Phi^{i+1} \leq \sup _{\mathbf{x} \in \partial \Omega, \mathbf{y} \in \partial \Omega}(K)\left(\frac{\beta^{i+1}}{\sigma(1-\beta)}\left(\int_{\Omega} \dot{q} d V+\int_{\partial \Omega} s d S\right)\right) \quad \text { on } \partial \Omega_{++}^{i+1}
$$

Since $T \geq \Phi^{i+1}$, it comes that

$$
T-\Phi^{i+1} \leq C\left(\sup _{\mathbf{x} \in \partial \Omega, \mathbf{y} \in \partial \Omega}(K)\right)^{1 / 4}\left(\frac{\beta^{i+1}}{\sigma}\right)^{1 / 4} \text { on } \partial \Omega_{++}^{i+1}
$$

and the supremum of $T-\Phi^{i+1}$ on $\partial \Omega_{++}^{i+1}$ is such that

$$
\sup _{\partial \Omega_{++}^{i+1}}\left(T-\Phi^{i+1}\right) \leq C\left(\sup _{\mathbf{x} \in \partial \Omega, \mathbf{y} \in \partial \Omega}(K)\right)^{1 / 4}\left(\frac{\beta^{i+1}}{\sigma}\right)^{1 / 4}
$$

Also,

$$
\begin{array}{r}
\sup _{\partial \Omega_{++}^{i+1}}\left(\omega-\Psi^{i+1}\right)=\sup _{\partial \Omega_{++}^{i+1}}\left(\int_{0}^{T} \hat{k}(\xi) d \xi-\int_{0}^{\Phi^{i+1}} \hat{k}(\xi) d \xi\right)=\sup _{\partial \Omega_{++}^{i+1}}\left(\int_{0}^{T} \hat{k}(\xi) d \xi\right) \leq \\
\leq\left(\sup _{\partial \Omega_{++}^{i+1}}\left(T-\Phi^{i+1}\right)\right)\left(\sup _{\Phi^{i+1}<\xi<T}(\hat{k}(\xi))\right) \leq\left(\sup _{\partial \Omega_{++}^{i+1}}\left(T-\Phi^{i+1}\right)\right)\left(\sup _{0<\xi<\infty}(\hat{k}(\xi))\right)
\end{array}
$$

and

$$
\sup _{\Omega}\left(\omega-\Psi^{i+1}\right)=\sup _{\Omega}\left(\int_{0}^{T} \hat{k}(\xi) d \xi-\int_{0}^{\Phi^{i+1}} \hat{k}(\xi) d \xi\right)=\sup _{\Omega}\left(\int_{0}^{T} \hat{k}(\xi) d \xi\right)
$$

Now, considering that

$$
\sup _{\Omega}\left(\omega-\Psi^{i+1}\right) \geq\left(\inf _{0<\xi<\infty} \hat{k}(\xi)\right) \sup _{\Omega}\left(T-\Phi^{i+1}\right)
$$

It may be concluded that

$$
\sup _{\Omega}\left(T-\Phi^{i+1}\right) \leq \frac{\left(\sup _{0<\xi<\infty} \hat{k}(\xi)\right)}{\left(\inf _{0<\xi<\infty} \hat{k}(\xi)\right)}\left\{\sup _{\mathbf{x} \in \partial \Omega, \mathbf{y} \in \partial \Omega}(K)\left(\frac{\beta^{i+1}}{\sigma}\left(\int_{\Omega} \dot{q} d V+\int_{\partial \Omega} s d S\right)\right)\right\}^{1 / 4}
$$

This could also be expressed as

$$
\left\|T-\Phi^{i}\right\|_{L^{\infty}(\Omega)} \leq \bar{C} \beta^{1 / 4}
$$

In (5.7) $\bar{C}$ represents a nonnegative constant, which is given by 


$$
\bar{C}=\frac{\left(\sup _{0<\xi<\infty} \hat{k}(\xi)\right)}{\left(\inf _{0<\xi<\infty} \hat{k}(\xi)\right)}\left\{\sup _{\mathbf{x} \in \partial \Omega, \mathbf{y} \in \partial \Omega}(K)\left(\frac{\beta^{i+1}}{\sigma}\left(\int_{\Omega} \dot{q} d V+\int_{\partial \Omega} s d S\right)\right)\right\}^{1 / 4}
$$

and \|\|$_{L^{\infty}(\Omega)}$ represents the norm of the supremum.

In order to emphasize the relevance of this result, it is important to notice that any real surface $K(\mathbf{x}, \mathbf{y})$ is bounded.

\section{Acknowledgement}

The authors gratefully acknowledge Brazilian agency CNPq for financial support.

\section{References}

[1] R.M.S. Gama, Existence, uniqueness and construction of the solution of the energy transfer problem in a rigid and non-convex blackbody with temperature- dependent thermal conductivity, Z. Angew. Math. Phys., 66 (2015), 2921-2939. doi:10.1007/s00033-015-0549-3.

[2] R.M.S. Gama, Numerical simulation of the (nonlinear) conduction/radiation heat transfer process in a nonconvex and black cylindrical body, J. Comput. Phys., 128 (1996), 341-350.

[3] R.M.S. Gama, J.A.O. Pessanha, J.A.R. Parise, F.E.M. Saboya, Analysis of a v-groove solar collector with a selective glass cover, Solar Energy, 36 (6) (1986), 509-519.

[4] F. John, Partial Differential Equations, Springer-Verlag, New York, 1971

[5] N. Afrin, Z.C. Feng, Y. Zhang, J. K. Chen, Inverse estimation of front surface temperature of a locally heated plate with temperature-dependent conductivity via Kirchhoff transformation, Int. J. Thermal Sci., 69 (2013), 53-60.

[6] S. Kim, A simple direct estimation of temperature-dependent thermal conductivity with Kirchhoff transformation, Int. Commun. Heat Mass Transfer, 28 (2001), 537-544.

[7] M.L. Martins-Costa, F.B.F. Rachid, R.M.S. Gama, An unconstrained mathematical description for conduction heat transfer problems with linear temperature-dependent thermal conductivity, Int. J. Non-Linear Mechanics, 81 (2016), 310-315. doi:10.1016/j.ijnonlinmec.2016.02.002.

[8] R.J. Moitsheki, T. Hayat, M.Y. Malik, Some exact solutions for a fin problem with a power law temperature-dependent thermal conductivity, Nonlinear Anal. Real World Appl., 11 (2010), 3287-3294.

[9] K.R. Rajagopal, G Saccomandi, Response of an elastic body whose heat conduction is pressure dependent, J. Elast., 105 (2011), 173-185.

[10] S. Chantasiriwan, Steady-state determination of temperature-dependent thermal conductivity, Int. Commun. Heat Mass Transfer, 29 (2002), 811-819.

[11] C.J. Glassbrenner, G.A. Slack, Thermal Conductivity of Silicon and Germanium from $3^{\circ} \mathrm{K}$ to the Melting Point, Phys. Rev., 134 (1964), $1058-1069$.

[12] Yu. Goldberg, M.E. Levinshtein, S.L. Rumyantsev, Properties of Advanced Semiconductor Materials GaN, AlN, SiC, BN, SiC, SiGe, M.E. Levinshtein, S.L. Rumyantsev, M.S. Shur (editors), John Wiley \& Sons, Inc., 2001, pp. 93-148.

[13] W. Joyce, Thermal resistance of heat sinks with temperature-dependent conductivity, Solid-State Electronics, 18 (1975), $321-322$.

[14] D. Tomatis, Heat conduction in nuclear fuel by the Kirchhoff transformation, Ann. Nuclear Energy, 57 (2013), 100-105.

[15] H.S. Carslaw, J. C. Jaeger, Conduction of Heat in Solids, Oxford, Clarendon, 1959.

[16] F. Incropera, P.D.Dewitt, Introduction to Heat Transfer, John Wiley \& Sons Inc.,1996.

[17] E.M. Sparrow, R.D. Cess, Radiation Heat Transfer, McGraw-Hill, 1978.

[18] V.S. Arpaci, Conduction Heat Transfer, Addison-Wesley Publishing Company, Inc., Massachusetts, 1966.

[19] M.S. Berger, Nonlinearity \& Functional Analysis: Lectures on Nonlinear Problems in Mathematical Analysis, Academic Press, London, 1977.

[20] A.E. Taylor, Introduction to Functional Analysis, Wiley Toppan, Tokyo, 1958. 